\title{
Die Union und ihre Nachbarn - Nachbarschaftspolitik vor und nach dem Verfassungsvertrag
}

\author{
Waldemar Hummer*
}

Gemäß Art. I-57 Abs. 1 des Vertrages über eine Verfassung für Europa ${ }^{1}$ (VVE) entwickelt die Union „,besondere Beziehungen zu den Ländern in ihrer Nachbarschaft, um einen Raum des Wohlstands und der guten Nachbarschaft zu schaffen, der auf den Werten der Union aufbaut und sich durch enge, friedliche Beziehungen auf der Grundlage der Zusammenarbeit auszeichnet“. In Ergänzung dazu verabschiedeten die Mitgliedstaaten in der Schlussakte von Rom die Erklärung (Nr. 11) zu Art. I-57, in der sie Folgendes postulierten: „Die Union trägt der besonderen Lage der Länder mit geringer territorialer Ausdehnung Rechnung, die spezifische Nachbarschaftsbeziehungen zur Union unterhalten."

Offensichtlich will die zukünftige Union sich damit zum einen werthaltungskonforme und sicherheitspolitisch relevante ,strategische Vormauern“ schaffen. Eine solche Bedeutung einer vertrauensbildenden Nachbarschaftspolitik - im Sinne eines so genannten ,ring of friends“ 3 von der Ostsee bis zum Mittelmeer - wird vor allem in der „Europäischen Sicherheitsstrategie - Ein sicheres Europa in einer besseren Welt" hervorgehoben, die der Europäische Rat von Brüssel am 12. Dezember 2003 auf Vorschlag des Hohen Vertreters für die GASP, Javier Solana, gebilligt hat. Darin wird als eines der drei strategischen Ziele der Union ausdrücklich die „Stärkung der Sicherheit in der Nachbarschaft“ erwähnt und der Europäischen Union diesbezüglich empfohlen, darauf hinzuarbeiten, ,dass östlich der EU und an den Mittelmeergrenzen ein Ring verantwortungsvoll regierter Staaten entsteht, mit denen wir enge, auf Zusammenarbeit gegründete Beziehungen pflegen können“"4. Zum anderen will die Union aber auch das Problem ihrer Überdehnung (over-expanding) ${ }^{5}$ durch weitere Beitritte in den Griff bekommen, indem sie ihren Nachbarstaaten durch eine neue Nachbarschaftspolitik eine attraktive Alternative zu einem Beitritt anzubieten versucht.

In welcher Grauzone man sich bewegt, wenn man Beitritts- und Nachbarschaftsaspirationen gegeneinander abzugrenzen versucht, zeigt folgende Aussage der Europäischen Kommission in ihrer einschlägigen Mitteilung „Größeres Europa - Nachbarschaft: Ein neuer Rahmen für die Beziehungen der EU zu ihren östlichen und südlichen Nachbarn“ auf: „In einigen Fällen ist die Frage einer möglichen Mitgliedschaft bereits gelöst worden. So ist ein Beitritt der nichteuropäischen Partnerländer im Mittelmeerraum ausgeschlossen worden.

* Ordentlicher Univ.-Prof. Dr. Dr. Dr. Waldemar Hummer, Leiter des Instituts für Völkerrecht, Europarecht und Internationale Beziehungen der Universität Innsbruck.

1 Unterzeichnet am 29. Oktober 2004 in Rom, CIG 87/2/04 Rev. 2, AB1. 2004, Nr. C 310, S. 1.

2 Die Erklärung (Nr. 11) bezieht sich auf die europäischen Kleinstaaten Andorra, Monaco, San Marino und den Staat der Vatikanstadt; vgl. dazu Waldemar Hummer: Die Kategorie des „Kleinst“- bzw. „Mikrostaates“ im Völkerrecht: Vom „Kleinststaat“ über den „künstlichen“ zum „virtuellen“ Staat, in: Erhard Busek/Waldemar Hummer (Hrsg.): Der Kleinstaat als Akteur in den Internationalen Beziehungen, Schaan 2004, S. 23-132.

3 Romano Prodi: Das größere Europa - eine Politik der Nachbarschaft als Schlüssel zur Stabilität. Rede vor der 6. Weltkonferenz des Studienverbandes der EG, Projekt Jean Monnet, Brüssel, 6. Dezember 2002, S. 3.

4 PESC 787, Dok. 15895/03, 8.12.2003, S. 10, abrufbar unter http://register.consilium.eu.int/pdf/de/03/st15/ st15895.de03.pdf (letzter Zugriff: 2.5.2005).

5 Michael Emerson: European Neighbourhood Policy: Strategy or Placebo?, CEPS Working Document No. 215, November 2004, S. 1. 
Andere Fälle sind jedoch noch nicht geklärt, z.B. die der europäischen Länder, die klar den Wunsch geäußert haben, der EU beizutreten." 6

Dieser ,,bewussten Ambiguität" ${ }^{\text {"7 }}$ der Kommissions-Mitteilung scheint sich auch das Europäische Parlament in seiner Entschließung vom 20. November $2003 \mathrm{zu}$ den „Beziehungen der Union zu ihren östlichen und südlichen Nachbarn"8 angeschlossen zu haben, führt es doch unter anderem zur gegenständlichen Abgrenzungsfrage - Beitritt versus Nachbarschaftsinstrument - Folgendes aus: Das Europäische Parlament ,geht davon aus, dass die Politik „Größeres Europa - Nachbarschaft" dem Bestreben einiger europäischer Nachbarstaaten um einen EU-Beitritt oder um unterschiedliche vertragliche Beziehungen keinesfalls entgegensteht, sondern dass sie - auch wenn sie mit der Erweiterungspolitik nicht deckungsgleich ist - ein wichtiges Instrument darstellen kann, um diese Länder in die Lage zu versetzen, auf der Basis der bis dahin erzielten Fortschritte einen Beitrittsantrag nach Art. 49 EUV zu stellen (...) “.9 Auf Grund dieser Unklarheit, die sogar die Organe in der Union nicht eindeutig lösen konnten oder auch wollten, ist daher zunächst der Abgrenzung zwischen Beitritt und sonstigen Formen privilegierter Partner- beziehungsweise Nachbarschaft nachzugehen.

\section{Beitritt versus privilegierte Partner- beziehungsweise Nachbarschaft}

Gemäß Art. 49 Abs. 1 EUV kann ,,jeder europäische Staat, der die in Art. 6 Abs. 1 EUV genannten Grundsätze achtet, beantragen, Mitglied der EU zu werden“. Damit gewährt Art. 49 Abs. 1 EUV jedem europäischen Staat (sofern er bestimmte Voraussetzungen erfüllt) das Recht, einen Antrag auf Beitritt zur Europäischen Union zu stellen - einen Rechtsanspruch auf Beitritt räumt er aber nicht ein, selbst wenn diese Voraussetzungen erfüllt sein sollten. Bemerkenswerterweise wird dieser Umstand nicht nur in der Lehre ${ }^{10}$ sondern auch von Organen in der Union selbst übersehen, stellte doch der Europäische Rat auf seiner Tagung in Luxemburg am 12. und 13. Dezember 1997 im Hinblick auf die Einberufung einer ,EuropaKonferenz" zur Erweiterung der Union fest, dass zu dieser Konferenz die Staaten eingeladen werden, die unter anderem anerkennen, ,dass jedes europäische Land, das die entsprechenden Kriterien erfüllt, berechtigt ist, der Europäischen Union beizutreten"“11.

Politisch bringen sich die Mitgliedstaaten der Union, und letztlich auch diese selbst, durch die Verleihung des Kandidatenstatus und die nachfolgende Aufnahme von Beitrittsverhandlungen mit einem Drittstaat zwar in Zugzwang, aber selbst positiv abgeschlossene Beitrittsverhandlungen gewähren nur ein Anwartschaftsrecht auf Beitritt und nicht ein Recht auf diesen selbst. Anderenfalls würde ja dem Beitrittswerber gegen den Staat, der durch eine Ratifikationsverweigerung des Beitrittsvertrages den Beitritt scheitern lässt, ein Schadensersatzanspruch zustehen. Die Aufnahme in die Europäische Union ist damit ein Akt, bei dem zwar eine Reihe (meta-)juristischer Beitrittsvoraussetzungen vorliegen müssen, der letztlich

6 KOM (2003) 104, 11.3.2003, S. 5; vgl. auch Marise Cremona: EU enlargement: solidarity and conditionality, in: ELR 1/2005, S. 13: „, (...) the new ENP is studiously neutral as regards the possible membership aspirations of some of the new neighbours".

7 Eckart Stratenschulte: Wandel durch Annäherung - oder Selbstaufgabe? Die Politik der ,neuen Nachbarschaft" und die Europäische Union, in: integration 1-2/2004, S. 95-100, hier S. 97; vgl. auch Eckart Stratenschulte: Das Brüsseler Illusionstheater - zu Gast in Osteuropa, in: Osteuropa 6/2003, S. 764-767.

8 Europäisches Parlament: Beziehungen der Union zu ihren östlichen und südlichen Nachbarn, P5_TAProv(22003)0520; Bericht des Ausschusses für auswärtige Angelegenheiten, Menschenrechte, gemeinsame Sicherheit und Verteidigungspolitik, A5-0378/2003, Berichterstatterin Pasqualina Napoletano.

9 Operativer Teil der Entschließung, P5_TA-Prov(22003)0520; A5-0378/2003, Punkt 14.

10 Vgl. Juli Zeh: Recht auf Beitritt? Ansprüche von Kandidatenstaaten gegen die EU, Schriften des Europa-Instituts der Universität des Saarlandes, Rechtswissenschaft, Band 41, Baden-Baden 2002.

11 BullEU 12-1997, S. 9 Ziff. I.5. 
aber im politischen Ermessen ${ }^{12}$ der für die Aufnahme zuständigen Organe - zum einen Rat und Europäisches Parlament gemäß Art. 49 Abs. 1 EUV und zum anderen alle EU-Mitgliedstaaten gemäß Art. 49 Abs. 2 EUV - liegt.

Was die Beitrittsvoraussetzungen betrifft, so muss es sich zunächst um einen europäischen Staat handeln, der auch die von Art. 6 Abs. 1 EUV geforderten Grundsätze beachtet. Des Weiteren muss er eine marktwirtschaftlich orientierte Wirtschaftsverfassung haben und auch in der Lage sein, den Acquis communautaire vollinhaltlich zu übernehmen. Dazu kommen noch die ursprünglich für die mittel- und osteuropäischen Staaten (MOEL) konzipierten weiteren Erfordernisse der so genannten „Kopenhagener Kriterien“ des Europäischen Rates von Kopenhagen vom Juni 1993. ${ }^{13}$

\section{Was ist ein ,europäischer Staat"“?}

Während sich alle anderen Voraussetzungen - vor allem aber die rechtsstaatlichen Grundsätze des Art. 6 Abs. 1 EUV - mehr oder weniger problemlos eruieren lassen, ${ }^{14}$ gestaltet sich die konkrete Auslegung des unbestimmten Vertragsbegriffs „europäischer Staat“ in Art. 49 Abs. 1 EUV mehr als komplex, war sie doch in der akademischen Diskussion bisher von der Dichotomie geografisches versus politisches ,Europa' geprägt.

In der einschlägigen neueren Literatur wird in diesem Zusammenhang darauf hingewiesen, dass es sich bei dieser regionalen Beschränkung der Beitrittsbewerber auf ,europäische Staaten" nicht nur um eine rein geografische Bestimmung des Begriffsinhalts handelt, sondern diesbezüglich auch der Nachweis historisch-kultureller und geistesgeschichtlicher Aspekte erbracht werden muss. Konsequenterweise kommen diese Autoren in der Folge zu dem Schluss, dass der in Art. 49 Abs. 1 EUV niedergelegte Beurteilungsspielraum im Falle der Türkei ,in durchaus problematischer Weise genutzt worden ist" ${ }^{" 15}$ - die Türkei sei nämlich weder in geografischer, noch in kulturell-geistesgeschichtlicher Hinsicht überwiegend europäisch.

Diese Argumentation ist nicht ganz überzeugend, kontrastiert sie doch mit einer weiteren denkmöglichen Interpretation des Begriffs ,,europäischer Staat“, der deswegen der Vorzug zu geben ist, da diese den Europäischen Rat nicht eines Ermessensmissbrauchs zeiht, als er auf seiner Tagung in Helsinki im Dezember 1999 der Türkei den Status eines Beitrittskandidaten eingeräumt hat. Geht man nämlich von den grundlegenden Werten der Rechtsstaatlichkeit und des Menschenrechtsschutzes in Art. 6 Abs. 1 EGV aus, dann sind dies genau dieselben Kriterien, die auch von der Satzung des Europarates - neben der Voraussetzung, ein ,europäischer Staat“" zu sein (Art. 4) - für eine Mitgliedschaft gefordert werden (Art. 3). Unter Berücksichtigung des Umstandes, dass Europarat und Europäische Gemeinschaften entstehungsgeschichtlich engstens zusammenhingen, ${ }^{16}$ und unter der Voraussetzung, dass sowohl die Mitgliedstaaten der Union als auch diese selbst die Satzung des Europarates - als Altvertrag im Sinne von Art. 307 EGV - zu berücksichtigen haben, kann die Zugehörigkeit zum Europarat als politischer Regionalorganisation in Europa durchaus als Abgrenzungskriterium

12 Matthias Pechstein: „Kaum justitiabler Beurteilungsspielraum“, Art. 49 EUV, Rdnr. 4, in: Rudolf Streinz (Hrsg.): EUV/EGV. Kommentar (2003), S. 203.

13 BullEG 6-1993, S. 13.

14 Vgl. dazu Waldemar Hummer: Vom Postulat ,struktureller Kongruenz und Homogenität“ zum (vertikalen) „Homogenitätsgebot“ des Art. 6 Abs. 1 EUV, in: Helmut Reinalter (Hrsg.): Die Zukunft der Demokratie, Innsbruck 2002, S. 151-176.

15 Pechstein: Art. 49 EUV, Rdnr. 3, 202.

16 Heinrich Schneider: Gründe für die Einrichtung von „Versammlungen“ bzw. „Parlamenten“ in (west)europäischen Organisationen: „Staatsrechtlicher Reflex“ oder ,demokratiepolitisches Feigenblatt“?, in: Erhard Busek/ Waldemar Hummer (Hrsg.): Etappen auf dem Weg zu einer europäischen Verfassung, Wien/Köln/Weimar 2004, S. 271-295. 
für die Bestimmung der Qualität eines „europäischen Staates“ auch im Sinne von Art. 49 EUV angesehen werden. Damit kann die Türkei, die - als Mitglied des Europarates - ohnehin als ,europäischer“ Staat rechtsstaatlich und grundrechtssichernd ausgestaltet sein muss, als Beitrittswerber zur Europäischen Union grundsätzlich nicht mehr diskreditiert werden. Das Argument, die Charakteristik eines ,europäischen Staates“ im Sinne von Art. 49 Abs. 1 EUV vor allem über dessen Mitgliedschaft im Europarat bestimmen zu können, wird auch dadurch gestärkt, dass es auf Grund fortschreitender Erweiterungen der Union zwischen den Mitgliedstaaten beider Organisationen - gegenwärtig sind 25 der 46 Mitglieder des Europarates auch Mitgliedstaaten der Europäischen Union - auch zu einer stärkeren Konvergenz kommen wird, was im Memorandum des Generalsekretärs des Europarates vom 25. September 2003 „One Europe - A Europe of Partners. Towards an associate partnership between the Council of Europe and the EU“17 folgendermaßen beschrieben wird: ,The Council of Europe has been and continues to be instrumental in preparing the applicant countries for accession to the EU (...) Council of Europe standards will be benchmarks for EU action within its Wider Europe strategy as well as in the development of its strategy towards the Western Balkans.“

Wird aber der Begriff eines ,europäischen Staates“ im Sinne von Art. 49 EUV vor allem über dessen Zugehörigkeit zum Europarat definiert, stellt sich sofort das Problem, dass damit alle 46 Mitgliedstaaten des Europarates zugleich auch als potenzielle Beitrittswerber der Union angesehen werden müssen. Dieser Vorstellung versuchte die Europäische Union bereits seit einiger Zeit gerecht zu werden, indem sie nämlich zwischen Staaten, die den Status eines Beitrittskandidaten erlangen können und solchen Staaten, denen diese Option nicht offen steht, differenzierte.

\section{Die EU als „hub-and-spoke“-Modell}

Für eine solche Klassifizierung entwickelte die Union auf der Basis eines „hub-and-spoke“Modells ${ }^{18}$ ein System ,konzentrischer Kreise“, in dem sie selbst als Zentrum, die Beitrittskandidaten als ,innerer Zirkel“ und die nicht-beitrittswilligen Drittstaaten als ,äußere Kreise“ figurierten. In diesem Modell fungierte die Europäische Union damit als Nabe (hub), die mit allen Drittstaaten durch von ihr ausgehende Speichen (spoke) verbunden war, wobei aber die Drittstaaten untereinander nicht durch eine Felge (rim) vernetzt waren. Damit saß die Union quasi wie eine ,Spinne im Netz', von der die bilateralen Präferenzen radial ausgingen beziehungsweise bei der diese konzentrisch zusammenliefen. Eine Verbindung der präferenzierten Drittstaaten untereinander im Sinne einer Felge sollte erst durch die so genannte ,paneuropäische diagonale Ursprungskumulierung “ erfolgen. ${ }^{19}$

In diesem Sinne unterteilte die Union ihre Nachbarstaaten in mehrere Gruppen, mit denen sie unterschiedliche Typen von Präferenzabkommen sowie Entwicklungshilfeprogrammen abschloss. Eine Gruppe stellten die EFTA-Staaten dar, denen die Europäische Union zum einen den multilateralen „Europäischen Wirtschaftsraum“ (EWR) (1994), zum anderen aber wie im Falle der Schweiz - eine Reihe bilateraler Sektorenabkommen anbot. Eine weitere Gruppe umfasste die mittel- und osteuropäischen Staaten (MOEL), die mit der Union durch bilaterale Europa-Abkommen sowie eine Reihe von Förderprogrammen - wie PHARE, SA-

17 SG/Inf(2003)35, abrufbar unter http://coe.int/t/e/sg/Secretary-General/Information/Documents/Numerical/ 2003/SGINF(2003)35E.asp (letzter Zugriff: 3.5.2005).

18 Vgl. dazu Waldemar Hummer: Die räumliche Erweiterung des Binnenmarktrechts, in: Armin Hatje (Hrsg.): Das Binnenmarktrecht als Daueraufgabe, EuR Beiheft 1-2002, S. 75-145, insbesondere ab S. 130.

19 Vgl. Hummer: Erweiterung des Binnenmarktrechts, S. 130. 
PARD und ISPA - verbunden waren. Für die Staatengruppe der westlichen Balkanländer entwickelte die Europäische Union so genannte „Stabilitäts- und Assoziationsabkommen“ (SAAs) und förderte sie durch das CARDS-Programm. ${ }^{20}$ Die GUS-Staaten erhielten von der Union bilaterale Partnerschafts- und Kooperationsübereinkommen (PKAs) und das TACISProgramm ${ }^{21}$ angeboten. Die zwölf Länder des südlichen Mittelmeeres erhielten durch die im Rahmen des so genannten Barcelona-Prozesses (1995) abgeschlossenen „Euro-Mediterranen Assoziierungsabkommen“ (EMAA) eine neue präferenzielle Stellung. Der Türkei²2 und Andorra $^{23}$ wiederum wurde eine bilaterale Zollunion konzediert. In diesem Sinne hatte die Europäische Gemeinschaft bis zum Jahre 2004 mehr als zwanzig bilaterale Assoziationsabkommen auf der Basis von Art. 310 EGV abgeschlossen.

In diesem Zusammenhang verdient der EWR am meisten Beachtung, wurde er doch im Januar 1989 von Kommissionspräsident Jacques Delors allein zu dem Zweck konzipiert, die beitrittswilligen EFTA-Staaten von diesem Schritt abzuhalten und sie in einem homogenen europäischen Wirtschaftsraum zweiter Geschwindigkeit ,einzuparken“, in dem allerdings „binnenmarktähnliche Verhältnisse“ herrschen sollten. Da sich die EFTA-Länder mit dieser zweitbesten Position aber nicht zufrieden geben wollten und trotz dieser Offerte weiterhin auf einem Beitritt beharrten und ihn letztlich auch durchsetzten, verlor der EWR auch für eine Reihe anderer Drittstaaten - vor allem für die MOEL - nicht nur seine Attraktivität als Ersatzlösung für einen nicht gewährten Beitritt zur Union, sondern auch als „Warteraum“ und „,wirtschaftspolitische Gehschule“ für zukünftige Beitrittskandidaten. ${ }^{24}$

Mit dieser Diskreditierung des EWR als allgemein anwendbares integrationspolitisches Modell ,zweiter Geschwindigkeit‘ sowie als Instrument eines wirtschaftspolitischen ,Probelaufs' für einen späteren Beitritt stand die Europäische Union vor dem Problem, über kein allgemein akzeptiertes Alternativmodell zu einem Beitritt zu verfügen. Sie sah sich daher unter anderem genötigt, eine „Neighbourhood Policy as Ersatz Enlargement“ ${ }^{“ 25}$ zu entwickeln. Parallel dazu sollte auch - mit dem neuen Ansatz eines ,Europa der Regionen “ - das zuvor dargestellte „hub-and-spoke“-Modell im Sinne konzentrischer Kreise durch ein Modell ineinander verwobener , Olympischer Ringe` ersetzt werden. Das Modell sollte nicht zu einer Abschottung der einzelnen Ringe untereinander führen, sondern vor allem die Staaten in den äußeren Zirkeln stärker mit den Zielen und Werten der inneren Ringe konvergieren lassen. ${ }^{26}$ Die Erweiterung um zehn neue Mitgliedstaaten zum 1. Mai 2004, die 2006/7 zu erwartenden weiteren Beitritte Rumäniens und Bulgariens sowie der Beitritt der Türkei und der westlichen Balkanstaaten - allerdings noch dies incertus quando - haben die Europäische Union nunmehr in Zugzwang gebracht, ihre bisherige Nachbarschaftspolitik zu überdenken und neu zu konzipieren. Um die Größenordnung der von der neuen Nachbarschaftspolitik umfassten Staatengruppe - der einstweilen keine Beitrittsoption eröffnet ist - zu dokumentieren, soll darauf hingewiesen werden, dass es sich dabei um sechzehn Staaten mit einer Einwohnerschaft von über 400 Millionen handelt. ${ }^{27}$

20 VO (EG) Nr. 2666/2000 des Rates vom 5.12.2000; AB1. 2000, Nr. L 306, S. 1-6.

21 VO (EG, Euratom) Nr. 99/2000 des Rates vom 29. Dezember 1999 (TACIS); ABl. 2000, Nr. L 12, S. 1-9.

22 Vgl. Hummer: Erweiterung des Binnenmarktrechts, S. $91 \mathrm{f}$.

23 Hummer: Die Kategorie des „Kleinst“- bzw. „Mikrostaates“ im Völkerrecht, S. 90 ff.

24 Eckart Stratenschulte: Ade, Ambiguität! Die neue Nachbarschaftspolitik der EU, in: Osteuropa 7/2004, S. 67: „Der EWR (...) taugt nicht als Leitstern“.

25 Marius Vahl: Models for the European Neighbourhood Policy. The European Economic Area and the Northern Dimension, CEPS Working Document No. 218/February 2005, S. 9.

26 Vahl: Models for the European Neighbourhood Policy, S. 1.

27 Vgl. dazu Michael Emerson: European Neighbourhood Policy: Strategy or Placebo?, CEPS Working Document No. 215/November 2004, S. 6, Tabelle 2; vgl. dazu nachstehend auf S. 195. 
Die Union, als Opfer ihres eigenen Erfolges, ist damit mit dem existenziellen Dilemma konfrontiert, ihre künftigen Grenzen zu definieren, ohne dabei ihre eigene Substanz und Identität zu verlieren. Sie ist dabei aufgefordert, „to do something really significant under the name of the new European Neighbourhood Policy, blurring the frontiers between ,in“ and „out", to the point that the Union might achieve beneficial leverage on developments in the periphery without rushing ahead with further accession negotiations"28.

Was die inhaltliche Ausgestaltung der neuen Nachbarschaftspolitik betrifft, so brachte es Kommissionspräsident Prodi mit folgender Formulierung ,sharing everything with the EU but institutions", ${ }^{29}$ auf den Punkt: Den Nachbarstaaten sollte wirtschaftlich weitestgehend entgegengekommen werden, ohne dass ihnen dabei aber eine Mitbestimmungsmöglichkeit durch Organteilnahme eröffnet werden sollte.

\section{Die neue Europäische Nachbarschaftspolitik}

Bereits im Vorfeld der fünften Erweiterung begann man in der Europäischen Union eine spezielle ,Europäische Nachbarschaftspolitik' (ENP) auszubilden, die die Kommission in ihrer Mitteilung „Europäische Nachbarschaftspolitik - Strategiepapier“ mit eigenen Worten folgendermaßen charakterisiert: „Die ENP wurde konzipiert, um der Zusammenarbeit mit den Nachbarländern der Europäischen Union nach der fünften Erweiterung neuen Schwung zu verleihen. Sie bereichert die Beziehungen zu den Partnerstaaten und knüpft dabei an die Erfahrungen bei der Unterstützung des Prozesses der politischen und wirtschaftlichen Transformation sowie an die wirtschaftliche Entwicklung und Modernisierung in den neuen Mitgliedstaaten und Kandidatenländern an. "30 Der entscheidende Passus ist dabei aber die Aussage, dass die ENP ,ein Instrument zur Stärkung der Beziehungen zwischen der EU und Partnerländern darstellt, das sich von den europäischen Ländern nach Artikel 49 EUV zur Verfügung stehenden Möglichkeiten unterscheidet“".31 Damit ist, wenngleich in verklausulierter Form, genau das ausgesagt, was vorstehend bereits angesprochen wurde, nämlich der Umstand, dass die ENP (nur) für die Nachbarstaaten entwickelt werden soll, denen keine Beitrittsoption eröffnet wird. Die ENP ist somit keine Vor-Beitrittsstrategie, sondern eine Politik, die auf eine dauerhafte und intensive Partnerschaft der Union mit Nicht-Beitrittsbewerbern ausgerichtet ist und deren Beitrittsgesuche verhindern soll.

Nach einem ersten gemeinsamen Schreiben des Hohen Vertreters für die GASP Javier Solana und des für die Außenbeziehungen zuständigen Kommissars Chris Patten vom August 2002 legte die Kommission am 11. März 2003 ihre Mitteilung „Größeres Europa - Nachbarschaft: Ein neuer Rahmen für die Beziehungen der EU zu ihren östlichen und südlichen Nachbarn“32 vor. Nachdem der Rat „Allgemeine Angelegenheiten und Außenbeziehungen“ diese Mitteilung in zwei Schlussfolgerungen vom 16. Juni 2003 und 13. Oktober 2003 begrüßt hatte, verabschiedete auch das Europäische Parlament am 20. November 2003 eine Entschließung zu den „Beziehungen der Union zu ihren östlichen und südlichen Nachbarn “33, in der es unter anderem die Nachbarschaftspolitik als geeignetes Alternativinstrument zu einem Beitritt zur Europäischen Union apostrophierte.

Im Unterschied zu den bisherigen Debatten wurden in der Mitteilung „Größeres Europa Nachbarschaft" die östlichen Nachbarn erstmals gemeinsam mit den Mittelmeeranrainern

28 Emerson: European Neighbourhood Policy, S. 1.

29 Romano Prodi: Das größere Europa, S. 5.

$30 \operatorname{KOM}(2004) 373$ endg., 12.5.2004, S. 6.

$31 \mathrm{KOM}(2004) 373$ endg., S. 2.

$32 \operatorname{KOM}(2003) 104$ endg., 11.3.2003.

33 Vgl. P5_TA-Prov(22003)0520; A5-0378/2003. 
behandelt, wenngleich in der Folge den westlichen Balkanstaaten, die in den ,Stabilisierungs- und Assoziierungsprozess der EU` (SAP) einbezogen sind und mit denen bereits spezielle SAAs - zur politischen Stabilisierung und zum Wiederaufbau, sowie zur nachhaltigen Entwicklung, Assoziierung und Integration derselben - abgeschlossen wurden, ${ }^{34}$ besondere „Europäische Partnerschaften“35 in Aussicht gestellt wurden.

\section{Die Länder des westlichen Balkans}

In der so genannten „Agenda von Thessaloniki für die westlichen Balkanstaaten“, die vom Rat ,Allgemeine Angelegenheiten und Außenbeziehungen“ auf seiner Tagung in Luxemburg am 16. Juni 2003 ausgearbeitet wurde, wird die europäische Perspektive der westlichen Balkanstaaten (Albanien, Bosnien-Herzegowina, Ehemalige Jugoslawische Republik Mazedonien, Kroatien, Serbien und Montenegro samt Kosovo) noch weiter ausgebaut und mit einer Beitrittsoption ausgestattet: „Die westlichen Balkanstaaten und die Unterstützung dieser Länder bei ihren Vorbereitungen auf eine künftige Integration in die europäischen Strukturen und letztlich den Beitritt zur Union haben für die EU hohe Priorität. Die Balkanstaaten werden integraler Bestandteil eines vereinigten Europas sein.“ Zugleich erinnert der Rat aber auch daran, dass alle westlichen Balkanstaaten Vertragsparteien sowohl des Internationalen Strafgerichtshofs und des Internationalen Gerichtshof für das ehemalige Jugoslawien (ICTY) sind und weist in diesem Zusammenhang darauf hin, dass ,die uneingeschränkte Zusammenarbeit mit dem ICTY, insbesondere hinsichtlich der Überstellung aller Angeklagten nach Den Haag und des uneingeschränkten Zugangs zu Unterlagen und Zeugen, somit entscheidend für eine weitere Annäherung an die EU [ist]" 36

Der Europäische Rat von Thessaloniki am 19. und 20. Juni $2003^{37}$ billigte diese Schlussfolgerungen des Rates und wies darauf hin, dass diese den Rahmen für deren Annäherung bis hin zu einem künftigen Beitritt bilden werden. Bereits einen Tag später, nämlich am 21. Juni 2003, kam es zum ersten „Forum EU-westliche Balkanstaaten“, dessen Schlusserklärung, zusammen mit der „Agenda von Thessaloniki“, die Grundlage für spätere Beitrittsverhandlungen bilden soll. Diesbezüglich wird für jedes Land des Westbalkans eine, europäische Partnerschaft" entworfen, die sich an den Beitrittspartnerschaften für die Bewerberländer orientiert und die an die Besonderheiten des SAP für Südosteuropa angepasst wird. Die spezifischen Kriterien des SAP wurden zunächst in den Schlussfolgerungen des Rates „Allgemeine Angelegenheiten“ vom 29. April 1997 zur Anwendung der Konditionalität bei der Entwicklung der Beziehungen zwischen der Union und bestimmten Ländern Südosteuropas $^{38}$ und danach in der Mitteilung der Kommission vom 26. Mai 1999 über den Stabilisierungs- und Assoziierungsprozess für die Länder Südosteuropas ${ }^{39}$ festgelegt. Der Rat über-

34 Für das SAA-Mazedonien siehe AB1. 2004, Nr. L 84, S. 13 ff. (in Kraft seit 1.4.2004), für das SAA-Kroatien $\operatorname{KOM}(2001) 371$ vom 9.7.2001 [vgl. dazu auch $\operatorname{KOM}(2004) 275$ vom 28.4.2004]. Mit Albanien wird seit Januar 2003 über den Abschluss eines SAA verhandelt, für Bosnien und Herzegowina liegt seit November 2003 eine Machbarkeitsstudie vor; vgl. auch KOM(2004) 202 vom 30.3.2004, S. 7 f.; siehe dazu auch Martin Brusis: Von der Ost- zur Südosterweiterung?, in: Osteuropa 11/2003, S. 1623-1638.

35 Vgl. dazu die VO (EG) Nr. 533/2004 des Rates, 22.3.2004, ABl. 2004, Nr. L 86, S. 1-2; zu den ersten von der Kommission vorgeschlagenen „Europäischen Partnerschaften“ siehe http://www.eu.int/comm/ external_relations/see/docs/index.htm (letzter Zugriff: 3.5.2005).

36 Vgl. Anlage A ,Agenda von Thessaloniki für die westlichen Balkanstaaten: Auf dem Weg zur Europäischen Integration" der 2518. Tagung des Rates - Außenbeziehungen -, 10369/03 (Presse 166), S. 11-19; laut Bericht der Chefanklägerin des ICTY, Carla del Ponte, an die Vereinten Nationen vom 9. Oktober 2003 und deren Ausführungen vor dem Rat „Allgemeine Angelegenheiten und Außenbeziehungen der EU“ am 13.10.2003 wird die Arbeit des ICTY in einigen Staaten des Westbalkans nach wie vor systematisch behindert.

37 Europäischer Rat (Thessaloniki): Schlussfolgerungen des Vorsitzes, 19./20.6.2003, Punkt 41.

38 BullEU 4-1997, Ziff. 2.2.1.

$39 \operatorname{KOM}(1999) 235$. 
prüft in speziellen Jahresberichten den SAP beziehungsweise die Mitarbeit der jeweiligen Länder an diesem gemäß dem so genannten „own merits approach“. Der Europäische Rat von Feira vom 20. Juni 2000 fügte den nunmehr so genannten „SAP-Kriterien“ zudem die volle Anwendbarkeit der „Kopenhagener Kriterien“"40 hinzu. ${ }^{41}$

\section{Die Ausgestaltung der neuen Nachbarschaftspolitik}

Neben dieser Beitrittsoption für die westlichen Balkanstaaten wies der Europäische Rat von Thessaloniki aber auch noch auf den Ausbau weiterer gutnachbarschaftlicher Beziehungen zu den durch die fünfte Erweiterung der Union als neue Nachbarn entstandenen sonstigen europäischen Staaten hin. ${ }^{42}$ Diesbezüglich hatte bereits der Europäische Rat von Athen vom 17. April 2003 erklärt, ,dass mit diesen neuen Nachbarn immer stärkere Bindungen und Brücken der Zusammenarbeit zu entwickeln sind und die Zukunft der Wertegemeinschaft der EU mit diesen Nachbarstaaten zu teilen ist"“.

Am 1. Juli 2003 legte die Kommission eine Mitteilung zur „Schaffung der Voraussetzungen für ein neues Nachbarschaftsinstrument "43 vor und richtete im Juli 2003 eine Task Force „Größeres Europa“ - unter dem Vorsitz von Kommissar Günter Verheugen - sowie eine dienststellenübergreifende Gruppe „Größeres Europa“ ein. Die dabei vorgeschlagene Methode besteht darin, zusammen mit den Partnern, die über geltende PKAs ${ }^{44}$ oder Assoziationsabkommen ${ }^{45}$ verfügen, Sondierungsgespräche einzuleiten, um - auf der Basis von speziellen Länderberichten der Kommission ${ }^{46}$ - eine Reihe von Prioritäten festzulegen, die anschließend in gemeinsame Aktionspläne ${ }^{47}$ aufgenommen und durch besondere Maßnahmen umgesetzt werden.

Die Aktionspläne werden von der Kommission in Abstimmung mit den betreffenden Ländern und mit dem Beitrag des Hohen Vertreters für die GASP zu Fragen, die die politischen Zusammenarbeit und die GASP betreffen, vorgelegt, und sollen in der Folge in den jeweiligen Räten der Partnerschafts- und Kooperationsabkommen sowie der Assoziationsabkommen genehmigt werden. Sobald die Prioritäten der Aktionspläne erfüllt sind, könnte der nächste Schritt die Aushandlung so genannter „Europäischer Nachbarschaftsabkommen“ (ENA) sein, die die bilateralen Abkommen der jetzigen Generation ersetzen könnten. ${ }^{48}$ Mit diesen ENAs ist realistischerweise erst zwischen 2007 und 2010 zu rechnen. ${ }^{49}$

Diese ,privilegierte Partnerschaft mit den Nachbarn" ${ }^{\circ 50}$ beruht auf einer gegenseitigen Verpflichtung auf gemeinsame Werte in den Bereichen Rechtsstaatlichkeit, good governance, Achtung der Menschenrechte, einschließlich der Minderheitenrechte, Förderung gutnachbar-

40 Vgl. BullEG 6-1993.

41 Europäischer Rat (Feira): Schlussfolgerungen, 20.6.2000; BullEU 6-2000, Ziff. I.49.

42 Europäischer Rat (Thessaloniki): Schlussfolgerungen des Vorsitzes, 19./20. Juni 2003, Punkt 44.

$43 \operatorname{KOM}(2003) 393$ endg. vom 1. Juli 2003.

44 Mit Armenien, Aserbaidschan, Georgien, Kasachstan, Kirgisistan, Moldavien, Russland, der Ukraine und Usbekistan; vgl. Hummer: Erweiterung des Binnenmarktrechts, S. 99.

45 Israel, Jordanien, Moldau, Marokko, Palästinensische Autonomiebehörde, Tunesien und Ukraine; vgl. Hummer: Erweiterung des Binnenmarktrechts, ab S. 119.

46 Am 12. Mai 2004 legte die Kommission die ersten ENP-Länderberichte für Moldawien, die Ukraine, Marokko, Tunesien, Israel, Jordanien sowie die Palästinensische Behörde für das Westjordanland und den Gazastreifen vor; SEK(2004) 564-570 vom 12. Mai 2004.

47 Erstmals im Dezember 2004 für die Nachbarländer Ukraine und Moldawien vorgelegt; vgl. KOM(2004) 795 endg. vom 9. Dezember 2004.

48 Europäische Kommission: Europäische Nachbarschaftspolitik. Strategiepapier, KOM(2004) 373 endg., 12.5.2004, S. 5

49 Stratenschulte: Ade, Ambiguität!, S. 70.

$50 \operatorname{KOM}(2004) 373$, S. 3. 
schaftlicher Beziehungen und Prinzipien der Marktwirtschaft sowie nachhaltige Entwicklung. Ferner wird ein Engagement für bestimmte wesentliche Aspekte des auswärtigen Handelns der Europäischen Union angestrebt, darunter insbesondere die Bekämpfung des Terrorismus und die Verbreitung von Massenvernichtungswaffen sowie die Einhaltung des Völkerrechts. Vor allem sollen die Nachbarstaaten durch die ENP aber auch in die Ausarbeitung eines ,Gemeinsamen Raums der Freiheit, der Sicherheit und des Rechts' eingebunden werden.

Da die neuen Nachbarstaaten die sie in absehbarer Zeit berührende Schengen-Außengrenze ${ }^{51}$ mit der Schaffung eines neuen „Eisernen Vorhangs“ gleichsetzen, ${ }^{52}$ spiegelbildlich dazu aber die EU-Mitgliedstaaten Fragen der Justiz und Inneren Sicherheit - innerhalb des Gemeinsamen Raums der Freiheit, der Sicherheit und des Rechts - zu den sensiblen Bereichen zählen, versucht die Nachbarschaftsstrategie beiden Interessenlagen durch ein effektives Grenz- und Visummanagement gerecht zu werden, in dem Sonderbestimmungen für den kleinen Grenzverkehr und vereinfachte Visumverfahren vorgeschlagen werden. Auf der anderen Seite will die Kommission die Zusammenarbeit in den Bereichen Migration, grenzüberschreitende Kriminalität und Asyl weiter verstärken.

\section{Die Finanzierung der neuen Nachbarschaftspolitik}

Sofort nach Abschluss der fünften Erweiterung implementierte die Kommission im Mai 2004 das Instrument einer europäischen Nachbarschaftspolitik, das sich aus folgenden drei Komponenten zusammensetzt: aus einem Strategiepapier, ${ }^{53}$ einzelnen Länderberichten ${ }^{54}$ und einem europäischen (finanziellen) Instrument für die Nachbarschaftspolitik. Letzteres, das im Rahmen der neuen Finanziellen Vorausschau 2007-2013 zur Anwendung kommen soll, wurde von der Kommission in zwei Mitteilungen ${ }^{55}$ erläutert und besteht aus vier Rechtsinstrumenten, von denen vor allem dem Vorschlag für eine „Verordnung mit allgemeinen Bestimmungen zur Schaffung eines Europäischen Nachbarschafts- und Partnerschaftsinstruments" (ENPI) ${ }^{56}$ besondere Bedeutung zukommt.

Das Europäische Nachbarschaftsinstrument ist ein neues Finanzinstrument, das sich der grenzüberschreitenden Zusammenarbeit widmet und zwar zusätzlich zu jenen Bereichen, die bereits durch vorhandene Nachbarschaftsprogramme - TACIS (für Russland und die westlichen Neuen Unabhängigen Staaten (NUS) $)^{57}$, MEDA (für die Mittelmeerländer) ${ }^{58}-$

51 Gem. Art. 3 Abs. 1 der Beitrittsakte (BA) (2003), AB1. 2003, Nr. L 236, S. 1 ff. ist der „Schengen acquis“, der durch das „Schengen-Protokoll“ (1997) in den Rahmen der EU übergeführt wurde - so wie er in Anhang I zur BA aufgelistet ist - ab dem 1. Mai 2004 von den neuen Mitgliedstaaten anzuwenden. Gem. Art. 3 Abs. 2 BA müssen die nicht in Anhang I zur BA genannten Bestimmungen des „Schengen-acquis“ für die neuen Mitgliedstaaten aber erst durch einen eigenen Ratsbeschluss für anwendbar erklärt werden; vgl. dazu allgemein Joanna Apap/Angelina Tchorbadjiyska: What about the Neighbours? The Impact of Schengen along the EU's External Borders, CEPS Working Document No. 210/October 2004.

52 Iris Kempe: Nachbarschaftspolitik: Russland, Ukraine, Moldau und Belaus, in: Werner Weidenfeld/Wolfgang Wessels (Hrsg.): Jahrbuch der Europäischen Integration 2003/2004, Baden-Baden 2004, S. 259-266, hier S. 261.

53 KOM (2004) 373.

54 Zeitgleich mit dem Strategiepapier legte die Kommission am 12. Mai 2004 Länderberichte zur Ukraine, $\operatorname{KOM}(2004)$ 373; SEK(2004) 566, und Moldau, KOM(2004) 373; SEK(2004) 567, sowie für die Mittelmeeranrainerstaaten, SEK(2004) 564-570, vor.

55 Mitteilung der Kommission „Unsere gemeinsame Zukunft aufbauen: Politische Herausforderungen und Haushaltsmittel der erweiterten Union - 2007-2013“, $\operatorname{KOM(2004)~101,~10.2.2004~und~} \operatorname{KOM}(2004)$ 487, 14.7.2004.

$56 \operatorname{KOM}(2004) 628$ endg. 29.9.2004.

57 TACIS steht für ,Technical Assistance to the Commonwealth of Independent States *

58 Unter dem MEDA-Programm (Mediterranean Development Assistance) erhielten die Mittelmeerländer in der Periode 1995-1999 3,424 Mrd. Euro und 5,350 Mrd. Euro für den Zeitraum 2000-2006; vgl. dazu die VO (EWG) Nr. 1762/92 des Rates, 29.6.1992, die VO (EG) Nr. 1488/96 des Rates, 23.7.1996 und die VO (EG) Nr. 2698/2000 des Rates, 27.11.2000; AB1. 1992, Nr. L 181, S. 1 ff., AB1. 1996, Nr. L 189, S. 1 ff., AB1. 2000, Nr. L 311, S. 1-8. 
abgedeckt werden. ${ }^{59}$ Neben den vier westlichen NUS und zehn Mittelmeerländern umfasst das ENPI auch noch die drei Länder des südlichen Kaukasus Armenien, Aserbaidschan und Georgien. Das ENPI richtet sich grundsätzlich auch an Russland, das aber beschlossen hat, seine strategische Partnerschaft mit der Europäischen Union nicht im Rahmen der ENP, sondern vielmehr mittels des Konzepts der ,gemeinsamen Räume“ zu entwickeln, wie nachstehend noch darzustellen sein wird.

Beim ENPI werden zwei getrennte Finanzierungsmechanismen zum Einsatz kommen: der Finanzierungsmechanismus 1 wird zur Förderung der grenzüberschreitenden Zusammenarbeit dienen, wobei die Gebiete an allen betreffenden Land- und Meeresgrenzen förderfähig sein werden. Es wird sich dabei in erster Linie um bilaterale Programme handeln. Im Gegensatz dazu dient der Finanzierungsmechanismus 2 der flexiblen Förderung einer breiter angelegten transnationalen Zusammenarbeit, an der sich Akteure und Begünstigte aus den Mitgliedstaaten und den Partnerländern beteiligen. ${ }^{60}$

Die Kommission hat in ihren Vorschlägen für die finanzielle Vorschau 2007-2013 das neue ENPI als eines der sechs Finanzinstrumente ${ }^{61}$ - mit einem Finanzvolumen von 14,029 Milliarden Euro - aufgenommen, die im Bereich der auswärtigen Beziehungen nach 2006 zum Einsatz kommen und die bestehenden Instrumente ablösen sollen.

Für ein solches Instrument mit doppeltem Charakter, das die beiden Bereiche ,Außenbeziehungen " und ,sozialer und wirtschaftlicher Zusammenhalt' (Kohäsion) innerhalb der Union abdecken und auf beiden Seiten der EU-Außengrenzen zum Einsatz kommen soll, gibt es im EG-Vertrag kein juristisches Präjudiz und damit auch kein sachliches Vorbild. Nach Auffassung der Kommission ist diesbezüglich Art. 181a EGV (,Wirtschaftliche, finanzielle und technische Zusammenarbeit mit Drittländern“) die angemessene Rechtsgrundlage, auf die auch der Rat seinen Beschluss 2005/47/EG vom 22. Dezember 2004 zur Änderung des Beschlusses 2000/24/EG im Hinblick auf die Erweiterung und die Europäische Nachbarschaftspolitik ${ }^{62}$ stützte. ${ }^{63}$ Durch diesen wurde eine Garantieleistung der Gemeinschaft für etwaige Verluste der EIB aus Darlehen für Vorhaben außerhalb der Gemeinschaft eingeführt und damit die Darlehensvergabekapazität der EIB unter anderem auch in den südöstlichen Nachbarländern sowie in den Mittelmeerländern gestärkt.

Die geografische Komponente der neuen Nachbarschaftspolitik: Europa-Kaukasus - Mittelmeer

Geografisch richtet sich die ENP an die bestehenden Nachbarn der Union - mit Ausnahme der westlichen Balkanstaaten - sowie an diejenigen Staaten, die infolge der fünften Erweiterung im Jahre 2004 näher an diese herangerückt sind. Räumlich exakt umschrieben ist die ENP aber nicht. ${ }^{64}$

59 Beide Programme liquidierten im Zeitraum 2000-2003 insgesamt 3,72 Mrd. Euro.

$60 \operatorname{KOM}(2004), 373$, S. 30.

61 Europäische Kommission: Unsere gemeinsame Zukunft aufbauen. Politische Herausforderungen und Haushaltsmittel der erweiterten Union - 2007-2013, KOM(2004) 101, 11.2.2004, S. 38.

62 AB1. 2005, Nr. L 21, S. 9 f.

63 Der Grundsatz-Beschluss 2000/24/EG des Rates vom 22. Dezember 1999 war noch auf Art. 308 EGV gestützt; AB1. 2000, Nr. L 9, S. 24-26.

64 Vgl. dazu Kempe: Nachbarschaftspolitik, S. 262: „Im Weiteren leidet das Konzept unter einer gewissen geographischen Beliebigkeit". 
In Europa gilt die ENP (nur) für folgende Staaten: Belarus, Moldawien, die Ukraine ${ }^{65}$ und den Sonderfall Russland ${ }^{66}$ - den anderen europäischen Staaten steht nach wie vor die Beitrittsoption offen.$^{67}$ In der Süd-Kaukasus-Region gilt die ENP für Armenien, Aserbaidschan und Georgien, mit denen die Europäische Gemeinschaft ebenfalls PKAs abgeschlossen hat. In der Mittelmeer-Region wiederum gilt die ENP für alle Teilnehmer am 1995 eingeleiteten so genannten ,Barcelona Prozess ‘ der ,Europa-Mittelmeer-Partnerschaft' (Algerien, Ägypten, Israel, Jordanien, Libanon, Marokko, Syrien, Tunesien sowie die Palästinensische Autonomiebehörde) - mit Ausnahme der Türkei. ${ }^{68}$ Die Europa-Mittelmeer-Partnerschaft erstreckt sich auf folgende drei Bereiche: Politik und Sicherheit, Wirtschaft und Finanzen sowie Soziales und Kultur. ${ }^{69}$ Dieser (bilaterale) Assoziierungsprozess wurde durch das so genannte ,Agadir-Abkommen' vom 8. Mai 2001 durch eine Freihandelszone zwischen Marokko, Tunesien, Ägypten und Jordanien horizontal ,multilateralisiert', indem eine entsprechende diagonale Ursprungskumulierung vereinbart wurde..$^{70}$

\section{Sonderfall Russland}

Obwohl Russland von der ENP teilweise mitumfasst ist, ${ }^{71}$ und im Rahmen des ENPI auch Finanzhilfe bekommt, nimmt es seine strategische Partnerschaft mit der Union - wie beim Gipfeltreffen am 31. Mai 2003 in Sankt Petersburg festgelegt - nicht auf dieser Basis, sondern durch die Errichtung von vier so genannten , gemeinsamen Räumen ' wahr: erstens ein gemeinsamer Wirtschaftsraum (unter besonderer Berücksichtigung von Umwelt und Energie); zweitens ein gemeinsamer Raum der Freiheit, Sicherheit und Justiz; drittens ein Raum der Zusammenarbeit im Bereich externe Sicherheit; und viertens ein Raum der Forschung und Bildung, einschließlich kultureller Aspekte. ${ }^{72}$ Auf dem Moskauer EU-Russland-Gipfel am 21. Mai $2004^{73}$ kam es auch schon - allerdings nur auf russischer Seite - zur Bestellung von vier Koordinatoren für diese gemeinsamen Räume. Des Weiteren einigte man sich auf einen Aktionsplan für einen „Gemeinsamen Europäischen Wirtschaftsraum“"74, wobei aber die Einbeziehung Russlands in den Binnenmarkt der Union - in einer EWR-ähnlichen Variante - auf breite Skepsis stößt. ${ }^{75}$ Russland seinerseits hat auf dem GUS-Gipfeltreffen in Jalta

$65 \operatorname{KOM}(2003)$ 104; die Ukraine (1998) und Moldawien (1995) streben einen Beitritt zur EU an; vgl. dazu Iris Kempe: Direkte Nachbarschaft. Die Beziehungen zwischen der erweiterten EU und der Russischen Föderation, Ukraine, Weißrussland und Moldova, Gütersloh 22000; Kempe: Nachbarschaftspolitik, S. 259 f.; vgl. den Aktionsplan und Zehn-Punkte-Plan der EU für die Ukraine, Agence Europe vom 26.1.2005.

66 Russland ist nicht in die „Europäische Nachbarschaftspolitik“ (ENP) einbezogen, sondern partizipiert nur am „Europäischen Nachbarschafts- und Partnerschaftsinstrument“ (ENPI) für die Finanzierung der grenzüberschreitenden Finanzierungsprogramme.

67 Vgl. Heinz Timmermann: Zur Strategie der EU gegenüber den Neuen Nachbarn Ukraine und Belarus, in: Osteuropa 11/2003, S. 1605-1622.

68 Vgl. dazu Hummer: Erweiterung des Binnenmarktrechts, S. $121 \mathrm{f}$.

69 Vgl. Michael Emerson/Gergana Noutcheva: From Barcelona Process to Neighbourhood Policy. Assessments and Open Issues, CEPS Working Document No. 220/March 2005, 1.

70 Vgl. Hummer: Erweiterung des Binnenmarktrechts, S. 123.

71 Vgl. dazu Fn. 66.

72 Vgl. die Mitteilung der Kommission über die Beziehungen zu Russland, KOM(2004) 106, 9.2.2004 sowie die Schlussfolgerungen des Rates, 24.2.2004.

73 IP/04/673, Brüssel, 21.5.2004.

74 Dieses Konzept wurde bereits beim EU-Russland-Gipfel im Mai 2001 aus der Taufe gehoben; vgl. Stratenschulte: Ade, Ambiguität!, S. 74; vgl. auch den Beschluss des Kooperationsrates zwischen den Europäischen Gemeinschaften und ihren Mitgliedstaaten einerseits und der Russischen Föderation andererseits vom 6. März 2002 zur Einsetzung einer gemischten hochrangigen Gruppe zur Ausarbeitung des Konzepts für einen gemeinsamen europäischen Wirtschaftsraum, AB1. 2002, Nr. L 82, S. 9-10.

75 Stratenschulte: Wandel durch Annäherung, S. 98. 
im September 2003 angekündigt, mit Belarus, Kasachstan und der Ukraine ebenfalls einen einheitlichen Wirtschaftsraum auszubilden.

Im unmittelbaren Vorfeld der Erweiterung wurde auch das PKA EU-Russland (1997) ${ }^{76}$ am 27. April 2004 durch ein Protokoll ergänzt, das die Ausdehnung desselben auf die neuen Mitgliedstaaten beinhaltet. Dazu kommt noch die spezielle Erleichterung im Transitverkehr zwischen Russland und der russischen Enklave Kaliningrad durch Litauen, ${ }^{77}$ sowie die Partizipation Russlands an Förderprojekten im Rahmen der so genannten „Nördlichen Dimension“78, die das Gebiet von Island bis Nordwestrussland und von der norwegischen See, der Barentssee und der Kara-See bis zur südlichen Ostseeküste umfasst, und instrumentell darauf abzielt, den besonderen Herausforderungen der Regionalentwicklung des nördlichen Europas gerecht zu werden. ${ }^{79}$

\section{Die Union und ihre Nachbarn im Verfassungsvertrag}

Wie vorstehend bereits erwähnt, entwickelt die Union gemäß Art. I-57 Abs. 1 VVE ,,besondere Beziehungen zu den Ländern in ihrer Nachbarschaft, um einen Raum des Wohlstands und der guten Nachbarschaft zu schaffen, der auf den Werten der Union aufbaut und sich durch enge, friedliche Beziehungen auf der Grundlage der Zusammenarbeit auszeichnet“. Diese Bestimmung enthält eine Reihe unbestimmter Begriffe, die auslegungsbedürftig sind, wie beispielsweise „,besondere Beziehungen“, „Länder in der Nachbarschaft der Union“, „gute Nachbarschaft“, „Raum des Wohlstands“, ,,enge Beziehungen“, „Grundlage der Zusammenarbeit" et cetera. Diese zum Teil programmatischen Formulierungen erhalten aber im Lichte der vorstehenden Ausführungen zur Europäischen Nachbarschaftspolitik beziehungsweise zum Europäischen Nachbarschaftsinstrument bereits gewisse Konturen. Im Grunde ist damit ein besonderes, enges Verhältnis zu den „Nachbarstaaten“ der Union angesprochen, das sich durch die Beachtung der in Art. I-2 VVE niedergelegten Werte der Union sowie durch friedliche, kooperative und gutnachbarschaftliche Beziehungen auszeichnet.

Laut Art. I-57 Abs. 2 VVE kann die Union spezielle Übereinkünfte mit den betreffenden „Nachbarstaaten“ im Sinne von Abs. 1 schließen, die ,gegenseitige Rechte und Pflichten umfassen und die Möglichkeit zu gemeinsamem Vorgehen eröffnen“. Diese Formulierung ist beinahe deckungsgleich mit der des Art. III-324 VVE, der den Abschluss von Assoziationsabkommen regelt, es fehlt lediglich der Hinweis auf das „,besondere Verfahren“ als immanenter Bestandteil eines Assoziationsverhältnisses. Dafür enthält Art. I-57 Abs. 2 VVE wiederum den Hinweis, dass ,,zur Durchführung der Übereinkünfte regelmäßige Konsultationen“ stattfinden. Letzterer Hinweis deutet wohl darauf hin, dass es sich bei den „Nachbarschafts-Übereinkommen“ um Abkommen handelt, die seitens der Union stärker überwacht werden und zu deren Implementierung Konsultationen notwendig sind. Wenngleich solche Konsultationen nicht mit einem ,Monitoring 'im engeren Sinne verwechselt werden dürfen, so haftet ihnen doch ein gewisser Anleitungs- und Kontrollcharakter an.

Ganz allgemein ist aber festzuhalten, dass sich die beiden Vertragskonstellationen in Art. I57 Abs. 2 VVE und Art. III-324 VVE schon alleine deswegen unterscheiden (müssen), da es in einem einzigen Vertrag für ,,idem“ aus rechtssystematischen Gründen keine unterschied-

76 AB1. 1997, Nr. L 327, S. 3 ff.

$77 \operatorname{KOM}(2001) 26$ endg., 17.1.2001; vgl. Hummer: Erweiterung des Binnenmarktrechts, S. 118 f.

78 Vgl. dazu z.B. den Beschluss des Rates 2001/777/EG, 6.11.2001; AB1. 2001, Nr. L 292, S. 41-42; vgl. Vahl: Models for the European Neighbourhood Policy.

79 Vgl. dazu auch die Ansätze im Rahmen des Council of Baltic Sea States (CBSS) sowie des Barents-Euro Arctic Council (BEAC) bei Nicola Catellani: The Multilevel Implementation of the Northern Dimension, in: Hanna Ojanen (Hrsg.): The Northern Dimension: Fuel for the EU?, Helsinki/Berlin 2001, S. 54-76. 
lichen Rechtsgrundlagen geben kann. Trotzdem muss die in diesem Zusammenhang getroffene Aussage, dass ,die Union mit dieser Bestimmung den Nachbarstaaten keine Assoziierungsperspektive und damit letztendlich auch keine Beitrittsperspektive einräumen“" wollte, ${ }^{80}$ relativiert werden. Eine so genannte „Beitritts-Assoziation“ im Sinne von Art. III324 VVE sowie ein „Beitritt“ im Sinne von Art. I-1 Abs. 2 VVE in Verbindung mit Art. I-58 VVE im engeren Sinn sind davon sicherlich nicht erfasst, eine assoziationsähnliche Verbindung im Sinne der so genannten „Hallstein-Formel“81 (gemäß derer ein Assoziationsverhältnis inhaltlich von einem einfachen präferenziellen Handelsabkommen , plus 1' bis zur Vollmitgliedschaft , minus 1' reichen kann) aber schon - und zwar auch in institutionalisierter Form.

\section{Zuständigkeit zur Konzipierung der Nachbarschaftspolitik}

Ressortmäßig nimmt innerhalb der am 19. November 2004 ernannten Europäischen Kommission für die Periode 2004-2009 Kommissarin Benita Ferrero-Waldner, neben den EUAußenbeziehungen ${ }^{82}$ auch die europäische Nachbarschaftspolitik wahr, wohingegen Kommissar Olli Rehn für die erweiterungsbedingten Fragen zuständig ist. Sollte der Verfassungsvertrag in Kraft treten, was frühestens am 1. November 2006 der Fall sein könnte, dann würde aber der bisherige Generalsekretär des Rates und Hohe Vertreter für die GASP, Javier Solana, das Amt des Außenministers der Union übernehmen ${ }^{83}$ und zugleich der Kommission als Vizepräsident angehören. Dann wäre freilich mit einer Neuverteilung der Aufgabengebiete zwischen ihm und der bisherigen Außenkommissarin Benita Ferrero-Waldner zu rechnen. In Verbindung damit ist eine neue Positionierung und Gewichtung der Nachbarschaftspolitik durchaus nicht ausgeschlossen.

\section{Einschätzung}

Ob die ENP alle die in sie gesetzten Erwartungen erfüllen wird, kann heute noch nicht genau beurteilt werden. Eines steht aber jetzt schon fest: ihre Funktion als Surrogat für einen nicht zugelassenen Beitritt zur Union wird von den europäischen Nachbarstaaten, denen keine Beitrittsoption eröffnet wurde (Ukraine, Moldawien, Georgien), in Frage gestellt werden, wie dies kürzlich nach dem Wahlsieg von Wiktor Juschtschenko im zweiten Wahlgang vom 26. Dezember 2004 in der Ukraine bereits geschehen ist. Sowohl der neugewählte Präsident als auch die Ministerpräsidentin Julija Timoschenko bestanden unmittelbar nach ihrer Vereidigung am 23. Januar beziehungsweise am 4. Februar 2005 darauf, dass die Ukraine von der Europäischen Union als Beitrittskandidat anerkannt und in die Reihe der Kandidatenstaaten aufgenommen wird. ${ }^{84}$ Der Erweiterungsdruck wird trotz Nachbarschaftspolitik daher anhalten.

80 Iris Kempe: Nachbarschaftspolitik: Russland, Ukraine, Moldau und Belarus, S. 260; Iris Kempe: Nachbarschaftspolitik als neues Handlungsfeld der EU, in: Werner Weidenfeld (Hrsg.): Die Europäische Verfassung in der Analyse, Gütersloh 2005, S. 250-258, hier S. 252; als CAP-Veröffentlichung auch online erhältlich unter http://www.cap.uni-muenchen.de/download/2003/2003_cd_eukonvent_kempe.pdf (letzter Zugriff: 17.5.2005).

81 Vgl. Michael Schweitzer/Waldemar Hummer: Europarecht, Neuwied 5 1996, S. 214 f. Rz. 703.

82 Vgl. dazu Waldemar Hummer (2005): Die Außenbeziehungen der Union, in: Christoph Vedder/Wolff Heintschel von Heinegg (Hrsg.): EU-Verfassung. Der Verfassungsvertrag. Kommentar, Baden-Baden 2005 (im Erscheinen).

83 Darauf haben sich die Staats- und Regierungschefs auf ihrem Sondertreffen in Brüssel am 29. Juni 2004 bereits verständigt.

84 Vgl. dazu den Aktionsplan und Zehn-Punkte-Plan der EU für die Ukraine, Agence Europe vom 26.1.2005, S. 7; vgl. dazu Barbara Lippert (2005): Die Türkei als Sonderfall und Wendepunkt der klassischen EU-Erweiterungspolitik, in: integration 2/05, S. 119-135, hier S. 134. 